\title{
EVALUACIÓN DE LA FORMACIÓN DEL PROFESORADO UNIVERSITARIO NOVEL
}

José Luís Medina Moya, Beatriz Jarauta Borrasca y Cecilia Urquizu Sánchez

Universidad de Barcelona, España

\section{INTRODUCCIÓN}

Durante el curso académico 2001-2002 se desarrolló en la Universidad de Barcelona el Curso de Postgrado "Innovación en la Docencia Universitaria" dirigido al profesorado novel de las facultades de Económicas, Derecho y Empresariales de la misma.

En el diseño ${ }^{1}$ del curso se contemplaba la realización de una evaluación interna y externa. Dentro de la primera se planteó, además de la evaluación de los aprendizajes y del programa realizados durante el transcurso del programa, la necesidad de conocer los efectos formativos posteriores en la práctica docente de los profesores noveles. Finalizado el programa se hacía imprescindible, por tanto, conocer su contribución a la mejora de la docencia del profesorado novel. El presente artículo recoge estos aspectos articulados en una evaluación diferida del impacto del Curso de Postgrado "Innovación en la Docencia Universitaria".

\section{FINALIDADES Y SENTIDO DE LA EVALUACIÓN}

A través de este estudio se pretende identificar y valorar los cambios, transformaciones y mejoras acaecidas en la práctica docente del profesorado novel como consecuencia de su participación en el Postgrado de "Innovación en la Docencia Universitaria." Se trata de conocer el "impacto", los "efectos" que ha tenido la formación ofrecida en su práctica docente. Sin embargo, la noción de "impacto" proveniente del mundo empresarial, que obedece a una lógica tecnocrática y mercantilista y se basa en una epistemología mecanicista, objetivante y reductora, no es la más adecuada para un fenómeno que, como el educativo, entendemos es de naturaleza compleja/ambigua, particular, fenomenológica y dialógica (Elliott. J., 1993). En efecto, desde el momento en que la docencia universitaria se entiende técnicamente, se está asignando a los procesos de enseñanza-aprendizaje una rigidez y previsibilidad de la que en realidad carecen (Alvarez, 1999). La enseñanza universitaria tiene lugar en espacios comunicativos en los que las interpretaciones son múltiples y a menudo conflictivas; donde existen diferentes orientaciones y expectativas de valor, saturados en suma, de indeterminación y ambigüedad (Medina, 1999). Esa variabilidad constante no se puede eliminar con la aplicación de reglas o prescripciones derivadas tecnológicamente so pena de distorsionar, hipersimplificando, una realidad compleja y multidimensional, una realidad que consiste en los significados

${ }^{1}$ El curso de Postgrado "Innovación en la docencia universitaria", con una duración de 168 horas distribuidas en 6 módulos teórico-prácticos y un proyecto final, era un curso presencial planificado para acceder a las bases fundamentales de la docencia universitaria del profesorado novel. El profesorado participante debía tener una experiencia entre 0 y 5 años de docencia en la universidad de Barcelona y desear obtener las competencias necesarias para la mejora de su práctica docente. El curso contemplaba un sistema tutorial basado en la mentorización del docente novel por parte de profesorado experimentado del departamento al cual pertenecía. Los asistentes al curso fueron 20 profesores y profesoras noveles. 
que los participantes en el acto educativo otorgan a sus conductas, o cayendo invariablemente en la trivialidad y la redundancia (Pérez Gómez, 1988).

Por otra parte los "posibles efectos" del curso de postgrado se articulan en lo que, en educación, denominamos "Cambio e Innovación." En este sentido, si se desea realizar un estudio de "impacto" y se parte del principio que el curso habría generado algunos procesos de cambio (todavía indeterminados) podemos estar realizando una atribución errónea: pensar que el cambio educativo obedece a una única causa o fuente de estimulo. Ciertamente, desde un punto de vista fenomenológico las causas y efectos de un cambio son imposibles de aislar. De esta manera, la evaluación de toda innovación educativa no debe ser vista como un mero tecnicismo descriptivo de resultados alcanzados, sino como la "transferencia" misma que un docente hace de toda situación de aprendizaje vivida. El manejar el término transferencia nos invita a ir más allá de las causas y efectos, es decir nos invita a aproximarnos al mundo de los significados y sentidos, a considerar la complejidad misma del aprendizaje desde la realidad compleja de cada sujeto. Llevar el término transferencia al campo de la evaluación nos permite ver si un programa de formación genera cambios y transformaciones reales en el sujeto y qué influencia tienen en su práctica docente (Fullan, 1991).

\section{CONTENIDOS, CRITERIOS Y METODOLOGÍA DE EVALUACIÓN}

Teniendo en cuenta la noción de impacto y transferencia más arriba descritos los contenidos de esta investigación evaluativa se refieren a las siguientes cuestiones:

- Los cambios cualitativos y/o cuantitativos que se están produciendo después de la formación recibida en el curso de Postgrado (conocimientos, habilidades, etc.).

- Las modificaciones (si se han producido y en qué sentido) en la comprensión que los docentes noveles tienen de su propia práctica.

- Las modificaciones y/o transformaciones de la práctica y su relación con esos cambios de visión y concepción

- Los cambios operados respecto a lo que se considera una "buena docencia"

- Las innovaciones y transformaciones metodológicas y los resultados obtenidos

- Las dificultades aparecidas durante la introducción de las innovaciones

En lo que se refiere a los criterios de evaluación, dos han sido los utilizados en este estudio: la utilidad percibida por el profesorado y la transferibilidad del conocimiento elaborado en el curso.

Dado que el objeto de la evaluación eran los cambios, transformaciones y mejoras en la práctica docente del profesorado novel tal y como ellos las percibían, se hacía necesario acceder a la comprensión que tenían los docentes de lo que había sido para ellos su proceso de formación. Para ello se optó por un enfoque metodológico cualitativo-fenomenológico (Van Manen, (2003) y por la técnica del grupo de discusión como los más apropiados para este estudio.

Durante el mes de marzo de 2003 se realizaron los dos grupos de discusión a los que asistieron 13 de los 20 docentes noveles convocados. La duración media de los grupos de discusión se situó en torno a las 3 horas. Las transcripciones literales de las dos sesiones fueron devueltas para su revisión y validación; 
cuando se nos retornaron recogimos todos los comentarios realizados por los entrevistados y los incorporamos a la nueva transcripción.

El sistema de notación que hemos utilizado para identificar los fragmentos de las transcripciones queda reflejado en el siguiente ejemplo:

“P3": donde "P" significa profesor/a, y "3" número de orden asignado para garantizar el anonimato.

\section{ANÁLISIS DE DATOS}

El método seguido para llevar a cabo el análisis de datos ha sido el de las comparaciones constantes propuesto por Glaser y Strauss (1967). Este es un método generativo, constructivo e inductivo en el que se combina la codificación inductiva de categorías con la comparación constante entre ellas. Una vez finalizado el análisis de los datos, obtuvimos la siguiente matriz dominios/metacategorías/ categorías:

\begin{tabular}{|c|c|c|}
\hline DOMINIOS & METACATEGORÍAS & CATEGORIAS \\
\hline \multirow{9}{*}{ CAMBIOS } & \multirow{3}{*}{$\begin{array}{l}\text { Conceptualización de } \\
\text { la docencia }\end{array}$} & Papel del profesor \\
\hline & & Finalidad de la enseñanza \\
\hline & & \\
\hline & \multirow[b]{2}{*}{ Cambios didácticos } & Estrategias didácticas \\
\hline & & Evaluación \\
\hline & & Resultados de aprendizaje \\
\hline & $\begin{array}{l}\text { Cambios en los } \\
\text { resultados }\end{array}$ & $\begin{array}{l}\text { Respuesta del alumnado } \\
\text { ante la innovación }\end{array}$ \\
\hline & \multirow[t]{2}{*}{ Valoración de cambios } & $\begin{array}{l}\text { Valoración de los cambios } \\
\text { PIDU }\end{array}$ \\
\hline & & $\begin{array}{l}\text { Valoración de los cambios } \\
\text { PIDU por parte de los } \\
\text { alumnos }\end{array}$ \\
\hline \multirow{14}{*}{\begin{tabular}{lrrr} 
FACTORES & \multicolumn{2}{c}{ OBSTACULIZADORES } & Y/O \\
INHIBITORIOS & DEL CAMBIO & Y & LA \\
INNOVACIÓN DOCENTE & &
\end{tabular}} & Didácticos & $\begin{array}{ll}\text { Problemas } & \text { didácticos } \\
\text { previos al PIDU } & \\
\end{array}$ \\
\hline & \multirow{3}{*}{ Institucionales } & Cultura académica \\
\hline & & $\begin{array}{l}\text { Falta de apoyo institucional } \\
\text { a la innovación }\end{array}$ \\
\hline & & Apoyo del departamento \\
\hline & \multirow[b]{2}{*}{ Recursos } & Falta de espacios \\
\hline & & $\begin{array}{l}\text { Falta de recursos } \\
\text { tecnológico-didácticos }\end{array}$ \\
\hline & \multirow[t]{2}{*}{ Organizativo } & $\begin{array}{lll}\begin{array}{l}\text { Aumento } \\
\text { dedicación }\end{array} & \text { tiempo } & \text { de } \\
\end{array}$ \\
\hline & & Grupos masificados \\
\hline & \multirow[b]{2}{*}{ Curriculares } & Rigidez curricular \\
\hline & & Diseño curricular \\
\hline & \multirow[t]{4}{*}{ Alumnado } & $\begin{array}{l}\text { Dificultad en la transferencia } \\
\text { y recontextualización del } \\
\text { contenido }\end{array}$ \\
\hline & & Resistencia del alumnado \\
\hline & & Expectativas del alumnado \\
\hline & & $\begin{array}{l}\text { Aumento de tiempo de } \\
\text { dedicación lumnado }\end{array}$ \\
\hline \multirow{6}{*}{ VALORACIÓN DEL PROGRAMA } & \multirow[b]{2}{*}{ Fortalezas } & Requisito para la docencia \\
\hline & & Experiencia positiva \\
\hline & \multirow{4}{*}{ Utilidad } & Utilidad Colateral \\
\hline & & $\begin{array}{l}\text { Fundamentación teórica de } \\
\text { la práctica }\end{array}$ \\
\hline & & $\begin{array}{l}\text { Mejoras en la comprensión } \\
\text { de la docencia }\end{array}$ \\
\hline & & Mejora de la práctica \\
\hline
\end{tabular}




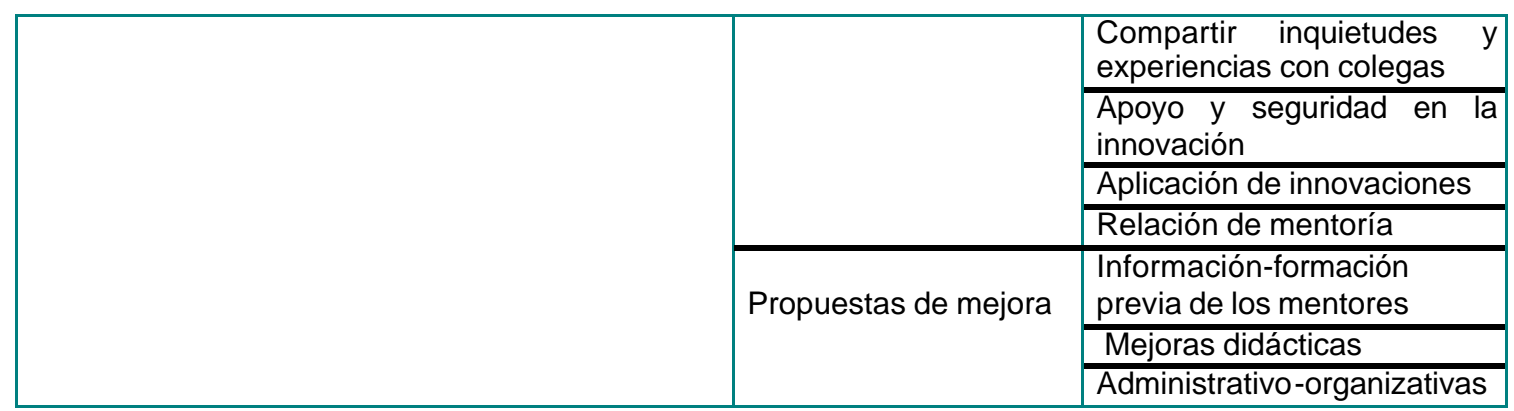

\section{RESULTADOS}

El siguiente esquema muestra los tres dominios cualitativos (y las metacategorías que los componen) que reflejan las percepciones y opiniones del profesorado respecto al programa en sí, a la contribución del curso a la mejora de su práctica docente y a las dificultades encontradas durante el desarrollo de las innovaciones.

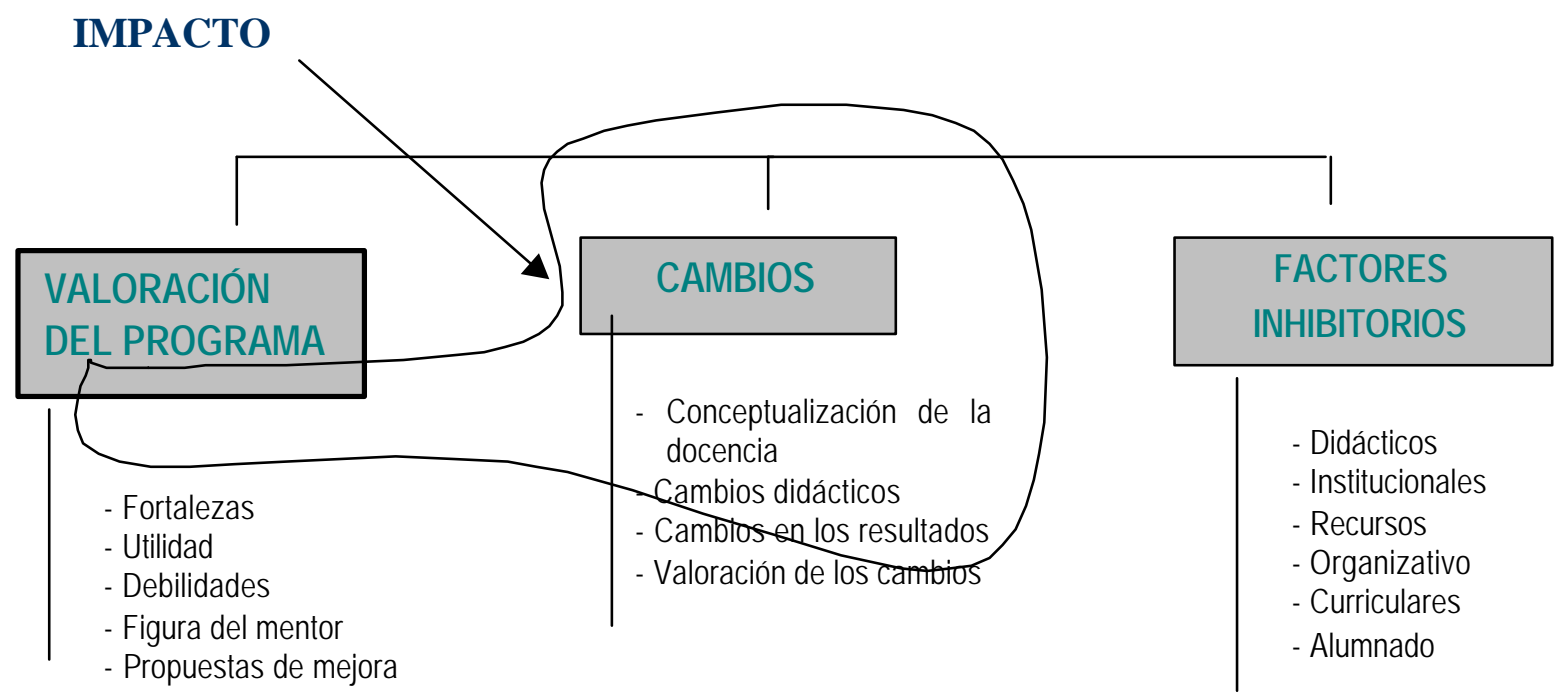

Nótese que esos tres dominios no son teórica ni empíricamente independientes entre sí. En primer lugar porque, tal y como se refleja en el esquema anterior, la transferencia y el impacto quedan substantivados en la utilidad percibida (dominio valoración del programa) por el profesorado y en los cambios e innovaciones realizadas. En efecto, durante el análisis de datos se ha considerado que la "Utilidad" del programa tenía entidad suficiente para ser considerada una metacategoría perteneciente al dominio "Valoración del Programa", aunque al mismo tiempo debe ser entendida como una parte de las fortalezas o efectos positivos del programa. Esa utilidad percibida por los profesores está íntimamente relacionada con los "cambios" acaecidos en su práctica docente. Por ejemplo, obtener una "fundamentación teórica de la docencia" o percibir "mejoras en la comprensión de la docencia" (categorías pertenecientes a "Utilidad") pueden conllevar a "cambios en la estrategias didácticas y de evaluación" (categorías pertenecientes al dominio "Cambios"). Todos estos aspectos quedan recogidos en las siguientes páginas. 
Por otra parte, es necesario matizar que los cambios que se han identificado en la práctica docente del profesorado novel no son un "efecto" "causado" exclusivamente por el curso. Ciertamente, es plausible pensar que el curso habrá tenido algunos efectos en la práctica docente del profesorado novel, pero esa práctica está determinada, también, por otros factores personales y contextuales además del curso, y ello imposibilita hablar del impacto o la transferencia del curso en términos de "causalidad lineal". Además, los cambios e innovaciones docentes que ha realizado el profesorado novel son, en parte, una expresión de la "aplicabilidad" de los aprendizajes realizados en el curso, entendida ésta como el grado en que los profesores noveles transfieren los conocimientos y habilidades adquiridos en las acciones formativas a su práctica docente o lugar de trabajo, la cual, es una mezcla compleja de factores personales, institucionales, organizativos, tecnológicos e ideológicos que pueden favorecer o dificultar la transferencia. El conocimiento de esos factores es una información de primera magnitud para poder introducir mecanismos de corrección que favorezcan la eficacia y eficiencia de la formación inicial del docente novel, esto es, que faciliten la introducción del cambio y la innovación. Por tanto, toda valoración del impacto de un programa de mejora de la docencia deberá tener en cuenta todas esas situaciones contextuales además de los resultados del programa. Es por este motivo que la tercera dimensión de los resultados que se presentarán hace referencia a los factores contextuales que dificultan y/o inhiben el cambio.

Tal y como puede observarse en la matriz de categorías/metacategorías/dominios, los aspectos emergentes (metacategorías) en los que se ha centrado el análisis y la evaluación del programa han sido sus fortalezas/utilidad, las debilidades o ámbitos a mejorar, la figura del mentor y las propuestas concretas de mejora realizadas por los participantes. Por razones de espacio no se presentarán en este trabajo los resultados referentes a los ámbitos y propuestas de mejora y a la figura del mentor. Nos centraremos exclusivamente en las fortalezas y la utilidad del programa que junto con los cambios introducidos en la práctica de los docente noveles conforman el impacto del curso de postgrado.

\subsection{La transferencia y el impacto del curso: fortalezas/utilidad/cambio}

En cuanto a las fortalezas del curso, el profesorado novel valora la participación en el curso de postgrado como una experiencia eminentemente positiva que debería convertirse en requisito para el ejercicio de la docencia:

P9. "Se podría hacer que cualquier profesor que quiera ser titular de universidad, tendría que entrar automáticamente en el curso.(...) debería haber una cláusula en la LOU, por la que cualquier profesor debería pasar por curso."

La percepción del profesorado acerca de la utilidad del curso de postrado, inicialmente genérica y poco articulada, se específica, y sustantiva en aspectos como la fundamentación teórica de la docencia, la mejora de la práctica, el apoyo en la aplicación de innovaciones, la mejora de la comprensión de la docencia - la ayuda para la realización del proyecto docente, que son las dimensiones constitutivas de la metacategoría utilidad y que a continuación se refleja:

a) Obtención de fundamentos y argumentos para teorizar la experiencia: hacia una profundización en la comprensión de la enseñanza universitaria. El profesorado novel afirma que el curso le ha ayudado a clarificar conceptualmente su trabajo docente. Esa clarificación conceptual, una vez organizada y articulada se ha constituido en el fundamento en el sustentar la práctica docente:

P4. "La base teórica sobre la que después poder apoyar la práctica docente tengo que manifestar que me la ha dado el postgrado y solo el postgrado (... ). “ 
Ese aumento de profundidad en la manera de entender el trabajo docente se ha substantivado también en un cambio de mirada hacia el alumnado. Pero ese cambio de mirada no es, de nuevo, una simple transformación en la comprensión conceptual, sino que transforma las relaciones didácticas que se dan en el aula. Unas relaciones que son ahora, más profundas, más significativas, esto es, más "pedagógicas" porque se fundan en una forma más adecuada de captar el significado que tiene el aprendizaje en la universidad para sus protagonistas, los estudiantes:

P4. "No me importa que no supieran cómo se hace un contrato, quizás, lo explico y ya está ,....) En ese aspecto si que me ha cambiado un poco, en no ser tan exigente con ellos y ser un poco más exigente conmigo misma (...) Repartir un poco la responsabilidad, que no sea responsabilidad de ellos, sino que sea una responsabilidad de los dos, por mi lado de ayudarlos a que la aprendan y por parte de ellos de aprender, no?"

El relato de esta profesora ilustra un hecho que, dada su obviedad, suele obviarse en la enseñanza universitaria: la relación existente entre enseñanza y aprendizaje no es lineal ni causal. En efecto, la tarea de la enseñanza universitaria no es "provocar" el aprendizaje (aunque esta sea su finalidad) sino permitir al estudiante realizar las tareas del aprendizaje que es consecuencia directa de su actividad de estudiar y no de la enseñanza.

c) Innovaciones docentes y mejora de la práctica

En resumen, la sistematización teórica lograda, el aumento de comprensión de la práctica y la reelaboración de la forma de entender el aprendizaje del alumnado han comportando llevar a la práctica innovaciones docentes:

P8. "He hecho nuevas innovaciones que a mí no se me habían ocurrido, alguna idea que he sacado de vosotros (...) sobre todo de estrategias y creo que me han servido bastante."

Innovaciones que han propiciado una cierta mejora de la práctica educativa:

P7. "En mi caso sí que la asistencia al postgrado me ha influido en lo que es la dinámica de la clase sobre todo, la gestión del aula (...) creo que es algo que me ha ayudado a mejorar mi práctica docente"

\section{d) La utilidad colateral}

Para terminar este apartado que hace referencia la utilidad que ha tenido el curso para los profesores asistentes, debemos mencionar sus "utilidades colaterales." Estamos aquí refiriéndonos a aquellos aspectos que ni en el diseño del curso se contemplaban explícitamente como intenciones, ni en su desarrollo se tematizaron en forma de contenidos, pero que, sin embargo, un elevado número de profesores han valorado positivamente.

En primer lugar, la ayuda que obtuvieron del curso para la elaboración de su proyecto docente realizado para presentarse a oposiciones:

P4. "Y a mí me sirvió mucho no sólo para la elaboración del proyecto docente sino que ahora me dices en que medida es el postgrado y en que medida es la reflexión personal del proyecto docente. Eso no lo sé pero ha sido muy positivo."

En segundo lugar, la influencia que en los mentores u otros colegas del departamento han podido tener los aprendizajes desarrollados por los profesores en el postgrado:

P1. "A través de nuestra experiencia el mentor se ha beneficiado de los conocimientos del postgrado, Es que no puedo decir otra cosa." 
Pero ha sido la experiencia de la elaboración del proyecto docente la que introduce una serie de cuestiones dignas de tener en cuenta. La elaboración del proyecto docente les ha exigido una "re-lectura" diferida y en otro "contexto motivacional" de algunas de las temáticas tratadas en el postgrado. Esta nueva situación, que exige la transferencia de aprendizajes al proyecto, ha suscitado en el profesorado la toma de conciencia de aspectos teóricos de su práctica docente hasta entonces desconocidos pero que se hallaban más o menos explícitos en los materiales didácticos aportados en el curso:

P5." Cuando estás elaborando un proyecto docente de alguna manera te sientas, te pones a pensar realmente lo que tú vas a hacer y con qué base. Yo me leí casi todos los apuntes del postgrado sino todos, pero hasta entonces no me había dado cuenta de las cosas, que ni se me habían pasado por la cabeza."

Es importante remarcar, sin embargo, que esos nuevos aprendizajes no tienen que ver solamente con la "necesidad" de realizar un proyecto docente, sino, y sobre todo, con la posibilidad de disponer de un tiempo de maduración y de reflexión sobre su propia práctica docente. Tiempos para la maduración de las ideas y espacios para la reflexión sobre la propia práctica emergen aquí como dos requisitos imprescindibles para una formación pedagógica del profesorado novel que aspire a ciertos niveles de calidad.

Los cambios acaecidos en la práctica docente del profesorado novel a partir de la experiencia desarrollada en el curso de postgrado han operado, mayoritariamente, en su conceptualización de la enseñanza, en sus estrategias docentes y de evaluación y en los resultados de aprendizaje. En lo que sigue se detallan todos estos aspectos:

\section{a) Otra manera de entender la enseñanza: finalidades, roles y relaciones}

Los cambios acaecidos en la forma de conceptualizar $^{2}$ y entender la docencia universitaria, se manifiestan por ejemplo, respecto a la finalidad de la misma:

P1. "He cambiado la forma de redactar las prácticas, ya no las hago tanto destinadas a vamos a descubrir dónde está el error o dónde está la norma que tengo que aplicar. Cuanto vamos a ver qué ideas te sugiere ese supuesto de hecho."

Esta profesora se refiere implícitamente a una de las cuestiones que más interés y controversia está suscitando en los últimos años en la enseñanza universitaria: la pregunta acerca del tipo de conocimiento que se requiere para un ejercicio profesional competente y virtuoso (en este caso el ejercicio de la abogacía). Hasta hace bien pocos años la respuesta dominante a esa cuestión se efectuaba en términos técnicos: se entendía el conocimiento profesional como un conjunto de hechos reglas y procedimientos que se aplican de forma no conflictiva a la resolución de problemas. Consecuentemente la formación consistía en ofrecer una serie de conocimientos teóricos para, después, aprender aplicarlos a situaciones previamente conocidas. Sin embargo, esta profesora constatando la ya amplia evidencia de que existen aspectos centrales en la práctica profesional (de la abogacía en este caso) que no pueden ser resueltos por la mera aplicación de reglas técnicas porque en esas actividades profesionales con frecuencia los problemas no aparecen bien delimitados ni claramente definidos, modifica sustancialmente las pretensiones de su enseñanza:

${ }^{2}$ Esta metacategoría (cambios/conceptualización de la docencia) aún teniendo similitudes con la categoría valoración del programa/utilidad/mejoras en la comprensión de la docencia la hemos diferenciado de aquella porque aunque entendemos que estos cambios en la conceptualización de la docencia implican una mejora de la comprensión de la misma, no han sido explícitamente así percibidos por los docentes noveles. 
P1. "Te das cuenta de que con la enseñanza no sólo les quieres impartir una determinada asignatura o unos conocimientos técnicos. Sino también, una forma de actuar."

Entiende que la formación técnica, aún siendo necesaria, asigna a la práctica profesional una certidumbre de la que realmente carece. Se trata entonces de ayudar al estudiante a resolver situaciones ambiguas y poco claras, tal cual se presentan en el ejercicio profesional y que requieren un conocimiento que va más allá de la mera capacidad de aplicación de reglas generales a situaciones particulares.

Otro de los cambios operados en la manera de entender la enseñanza se relaciona con la reconceptualización del papel del profesor universitario y con sus relaciones con el alumnado. Se observa un tránsito de una concepción del docente como transmisor de conocimientos a una visión centrada en la facilitación y personalización del aprendizaje. Un docente que fomenta ya el aprendizaje significativo y comprensivo antes que el memorístico, que no sólo instruye en el aula, sino que orienta y ayuda fuera de ella:

P8:" Yo intento que no se aprendan nada de memoria sino que me den una deducción lógica, intento que además haya problemas que entiendan a la primera, de atrás hacia adelante, me da igual el orden mientras lo razonen (...),bueno, memorizar ya lo mínimo posible."

P6. "Creo que forma parte de mi trabajo ayudarlo, y asesorarlos y eso. Que pasa que los escucho y el trabajo del profesor no sólo es la clase, su asignatura."

b) Los cambios e innovaciones didácticas: las estrategias de enseñanza y la evaluación de aprendizajes

Esas transformaciones en la conceptualización de la finalidad de la enseñanza y en la comprensión del papel del profesorado han tenido su correlato en el aula, sobre todo, en cambios en las estrategias de enseñanza aprendizaje y en las modalidades de evaluación.

P2. "El cambio más evidente para mí, es que he empezado a repartir papelitos entre los estudiantes, que no lo había hecho nunca de verdad, eh?. O cuando les explicaba el derecho en la contabilidad lo que hacía es empezar con un par de chistes proyectados en transparencias. Este es el cambio más inmediato que yo puedo decir que veo en mi práctica docente inmediata."

P8. "Uno de los exámenes lo he hecho vía casos es decir ya no pregunto qué epígrafe 2,3,4."

Pero a ese cambio en las estrategias de la evaluación de acreditación o sumativa hemos de añadir también el operado en la propia concepción del sentido de la evaluación que se manifiesta en un aumento de importancia de la evaluación formativa:

P4. "Porque yo estoy intentando, darle un poco de peso a la evaluación continua de alguna manera pues para compensar."

A la hora de valorar en su justa medida este cambio, hemos de tener en cuenta que el profesorado novel partícipe del postgrado forma parte de facultades con una fuerte tradición en evaluación sumativa, es decir, los aprendizajes realizados por el alumnado se evalúan, fundamentalmente, a través de un examen final. Por lo tanto, el hecho de introducir cambios en la evaluación ha supuesto una profunda innovación y una verdadera ruptura con los sistemas tradicionales de evaluación a los que tan acostumbrados están los profesores y alumnos de estas facultades.

\section{c) Las relaciones profesorado-alumnado}

Pero esos cambios en la conceptualización de la docencia que se han substantivado en innovaciones didácticas y evaluativas sólo adquieren pleno sentido en relación con el otro protagonista del proceso enseñanza-aprendizaje: el alumnado y con la finalidad última de ese proceso, o sea, el aprendizaje 
del alumno. Las relaciones entre profesorado y alumnado que antes podían ser caracterizadas como distantes y jerárquicas, parece que ahora son menos estereotipadas, más personalizadas y próximas:

P4. "Yo creo que hay más interacción entre yo que estoy en la tarima y ellos que están abajo. Pues ahora yo suelo bajar mucho abajo. En mis movimiento de clase yo también lo he notado. Eso de estar explicando y bajar donde están ellos y un poco pues acercarse a ellos, incluso a veces tocarlos."

Sin embargo, relaciones más próximas y cordiales abren la puerta a una situación que el profesorado percibe como problemática. Tal y como esta profesora relata, cuando el alumno se muestra como sujeto más allá de los estereotipos al uso, suelen generarse situaciones dilemáticas y problemáticas que desbordan al docente y que pueden acabar frustrándolo:

P1. "Cuando llegas a un contacto mas personal con el alumno, no sólo son los problemas de la asignatura los que recaen sobre ti, sino que a veces los alumnos cosas que le iban a decir al jefe de estudios, te viene y te cuentan (...)Que te vienen a contar problemas al margen de la asignatura. Entonces esto acaba entonces por frustrar."

Pero la enseñanza universitaria tiene lugar en espacios comunicativos en los que las interpretaciones son múltiples y a menudo conflictivas, donde existen diferentes percepciones de situaciones semejantes, propiciadas por la existencia de distintas expectativas de valor. Todo ello hace que esas mismas situaciones son también percibidas de manera ambivalente por el profesorado. Por un lado, el profesorado no se siente preparado para afrontarlas y al tiempo suponen un enriquecimiento en su relación con el alumnado:

P1. "Estos casos que tú mismo te sientes que no sabes como actuar. Tienes una persona con un determinado problema y no sabes ni a dónde orientarle porque ves que excede de lo que son, de lo que es tu responsabilidad como profesor. Era una persona que no sé, con, con otro órgano de la facultad, pero no contigo como profesor, pero la relación profesor-alumno se enriquece sobre manera, es, es lo que más ha cambiado."

Las reflexiones de estas profesoras ilustran la "hiperracionalización" de la docencia universitaria en la que se han excluido cualquier alusión a las dimensiones, sociales, afectivas y morales que concurren en todo proceso de aprendizaje. Ciertamente, el aula universitaria es "el lugar" en el que se realizan las actividades de aprendizaje; pero también es buena parte de "lo que se aprende", porque es un espacio físico, pero también social, en el que las relaciones entre las personas se desarrollan de acuerdo con patrones que implícita o explícitamente hacen referencia a valores. Las experiencias que se desarrollen en el aula no pueden pensarse solamente como "un" medio para alcanzar determinadas metas, sino como "el" medio en el que se viven experiencias en sí mismas deseables. Todo método pedagógico conlleva un determinado clima social el cual también es aprendido. Modelos pedagógicos que suponen grupos democráticos crean sistemas sociales democráticos y exigen que los alumnos aprendan (además de los contenidos de que se trate) valores como la solidaridad y el respeto. Esto significa que la clase debe constituirse como el lugar para experimentar y practicar los contenidos de la propia asignatura, teniendo la posibilidad de analizarla e interpretarla, de examinar sus propuestas y de decidir sobre su evolución. 
d) Las respuestas del alumnado y su aprendizaje

Decíamos en los epígrafes anteriores que los cambios e innovaciones docentes allí relatados cobran sentido únicamente en relación con el otro protagonista del proceso de E-A: el alumnado ${ }^{3}$.

La relación más cercana y personalizada que ha fomentado la realización del curso no sólo ha generado dilemas en el profesorado sino que también ha tenido efectos positivos en alumnado y sus relaciones con el docente. En primer lugar, podría hablarse de un cierto goce y satisfacción durante el trabajo de aula:

P3. "Los alumnos han reaccionado muy bien a todos los recursos y todas las prácticas que he implantado (...) los alumnos muy satisfechos lo pasamos muy bien en clases.(...) Es que el alumno como mínimo está a gusto en tu clase, que esto es una satisfacción añadida, no?."

Este cambio en la actitud del alumnado que ahora se convierte, mediado por el cambio de actitud del docente, en protagonista activo de su propio proceso de construcción de conocimientos tiene una influencia clara en los resultados de aprendizaje, resultados que, según el profesorado novel, se derivan de una forma más autónoma, reflexiva y significativa de aprender y que, por tanto, implican una mejora cualitativa del aprendizaje:

P5. "Por lo que yo veo en la evaluación continua yo que estoy con ellos, que comento los problemas, yo creo que si que ha habido una mejora del aprendizaje."

e) La valoración del cambio por parte del profesorado

Sin embargo, esa mejora cualitativa, parece que, según el profesorado novel, no se refleja en los resultados cuantitativos de la evaluación sumativa o final:

P5." Lo que vengo a decir es que hacer un poco todo este tipo de actividades y de cosas, por lo menos en mis experiencias no ha afectado los resultados de los exámenes".

Pero, aunque el profesorado observa que los resultados finales son semejantes con el nuevo sistema de trabajo, aceptan que, paradójicamente, existe una mejora en el aprendizaje:

P5. "En la evaluación continua yo creo que si que ha habido una mejora del aprendizaje, lo que no se es porque no lo demuestran."

Mejora debida, según ellos, a que la calificación no es ya únicamente la puntuación obtenida en el examen final sino que a ésta se añade la obtenida en la evaluación continua:

P5. "Sí claro. Lo que yo te digo es que la mejora que yo he encontrado es debida a que ellos hacen una evaluación continua y eso les puntúa, los puntúa de una manera muy positiva de cara al examen. Lo que no he encontrado mejora es en la evaluación del examen en sí."

Mejora que es de nuevo relativizada cuando se la sitúa en el contexto de los resultados cuantitativos finales:

P5. "Que el número de aprobados haya sido mayor no significa que los exámenes hayan estado mejor. Ha significado que han hecho una evaluación continua y han obtenido una serie de puntos que le ha ayudado a mejorar la nota del examen. Entonces aprueban peor lo que es el examen como tal, en si la gente no lo está haciendo mejor."

\footnotetext{
${ }^{3}$ La imposibilidad material de acceder directamente al alumnado, ha obligado a conocer sus opiniones y experiencias de manera diferida a través de las opiniones del profesorado novel.
} 
¿Cómo podríamos explicar esta aparente contradicción? Según el profesorado los resultados que obtienen los alumnos con la introducción de las innovaciones son los mismos en términos de notas pero probablemente no lo sean en términos de aprendizajes. Quizás el problema puede hallarse en la prueba final. Si como se deriva de la opinión de los profesores, es plausible inferir que se han producido mejoras en el aprendizaje, cabe preguntarse si la prueba final es sensible a esos cambios y puede medir esa mejora.

Parece también que algunos de estos profesores dan una importancia desmedida a la nota del examen porque creen que allí se refleja unívocamente el grado de logro de aprendizaje y porque la sobrevaloran, como indicador de aprendizaje por encima de la información que obtienen de la evaluación formativa. Y aquí puede residir, quizás, otro de los factores que expliquen esta aparente paradoja: la posible confusión entre evaluación formativa y calificación.

El problema de la evaluación en la universidad es que los docentes deben desarrollar simultáneamente la función de acreditación y la de evaluación, lo que facilita su confusión que, en ocasiones, se transforma en una verdadera contradicción.

\subsection{Factores inhibidores del cambio y la innovación}

Todos los cambios que hasta aquí se han ilustrado no se han producido, obviamente, en un vacío social o institucional. Tal y como ya se ha remarcado las innovaciones docentes tienen lugar en el seno de la institución universitaria que constituye una red compleja de factores interrelacionados que pueden fomentar o dificultar el cambio y la innovación docente. Las innovaciones docentes que el profesorado ha emprendido a partir del programa se han visto inhibidas por factores didácticos, institucionales, organizativos, curriculares, y obstaculizadas por cuestiones relacionadas con la falta de recursos y con las características del alumnado.

a) Los difíciles inicios: la inseguridad del docente novel

El primer factor que dificulta o impide la innovación docente es la falta de seguridad en la práctica docente:

P.8. "Cuando tienes tanta inseguridad te alejas ${ }^{4}$ ¿no? Porque no conoces claro y no te metes allí porque claro, que te van a decir, no sé qué, sino sabes esto como vas a reaccionar si te preguntan una cosa."

La sensación de inseguridad que experimenta el docente novel se debe, sobre todo a una falta de experiencia y de conocimiento pedagógico. Esta inseguridad la entienden como una consecuencia de su incipiente dominio en métodos, técnicas y recursos de enseñanza:

P.8: "Notaba una inseguridad (...) El programa y todo los recursos me dieron un poco más de seguridad en la aplicación de las técnicas y de los recursos."

Es importante remarcar aquí que ese proceder titubeante pleno de perplejidades es un "rasgo propio" de los principiantes y que debe ser interpretado no como una anomalía a ser rápidamente resuelta sino como una reacción natural y coherente que debe ser objeto de consideración y reflexión como parte necesaria del proceso de desarrollo profesional del docente universitario.

\footnotetext{
${ }^{4}$ En el sentido de baja implicación.
} 
b) Espacios insuficientes y recursos tecnológicos escasos

Otro de los factores que obstaculizan la innovación docente, según los profesores noveles, es el déficit de recursos materiales disponibles en la universidad. En primer lugar, espacios inadecuados que imposibilitan la realización de actividades y prácticas innovadoras en el aula:

P1: "Básicamente las dificultades eran las instalaciones, las clases en derecho, pues han de ser parecidas a las vuestras, lo que pasa es que las salas no son inclinadas son rectas."

En segundo lugar, la inexistencia de espacios que favorezcan el trabajo en grupo del alumnado:

P1 :" Otro problema más es que los alumnos no tienen sitio para reunirse, para hacer grupos."

La falta de recursos tecnológicos también es considerada como un gran inconveniente para promover el uso de las TIC (Teconologías de la Información y la Comunicación) como soporte del proceso de enseñanza-aprendizaje:

P2: "Tampoco hay muchos ordenadores...no todo el mundo...eh!."

c) La dimensión organizativa: grupos masificados y la problemática del tiempo

Uno de los factores que más han dificultado la puesta en práctica de las innovaciones docentes surgidas en el seno del curso ha sido el elevado número de alumnos que tienen por asignatura:

P3. "Nos costó, porque claro con cien alumnos, qué haces, no?. Vamos a hacer un juego,... si te despistas, bueno eso era un gallinero, no?."

Pero esa dificultad no sólo se ha experimentado en aulas masificadas a la hora de implantar estrategias didácticas que fomenten el trabajo autónomo del alumnado. También fuera de ella el aumento de tiempo de dedicación que generaba la puesta en marcha de la innovación ha sido considerable:

P2. "Hay otro elemento, el elemento tiempo que nosotros lo vemos al organizarlo, tiene mucho más trabajo."

\section{d) El seno departamental y las culturas académicas}

El profesorado participante en el curso cree que su elevada dedicación a la docencia y el exceso de tiempo que invierte en la innovación docente, en ocasiones, no es reconocida suficientemente por el departamento al que pertenece:

P4. "El departamento vivía al margen de esta experiencia. El departamento es así. Hay algo que falta."

Bajo interés que no es una consecuencia de las opiniones que pueda suscitar la valoración de la innovación que llevan a cabo los docentes noveles, sino que está más relacionado con la tradicional cultura académica ${ }^{5}$ universitaria individualista, de aislamiento y con límites muy bien definidos entre grupos docentes:

P2. "Me he acogido a la cultura departamental de que nadie se mete con nadie."

Ese desinterés es también una muestra específica de la todavía baja consideración que recibe la docencia en el curriculum y en el desarrollo profesional del profesorado universitario:

\footnotetext{
${ }^{5}$ Por cultura académica entiéndase aquí el conjunto de creencias, valores, normas y hábitos que constituyen el marco interpretativo a la luz del cual adquiere sentido y significado la práctica docente al tiempo que configuran la identidad de los docentes y del departamento o facultad y definen las relaciones que mantienen entre sí los docentes. Ese sistema de creencias y hábitos se elabora de manera colectiva por los grupos docentes que durante años se enfrentan al mismo tipo de realidades, dificultades y situaciones.
} 
P8. "Es un departamento que hay muchísima gente joven y no, no porque además ahora están valorando mucho la investigación, entonces claro la docencia dicen la docencia da igual no me van ni a dar más puntos por docencia ni, simplemente es investigación."

Sin embargo, esta opinión es matizada por la experiencia de otros profesores noveles que sí han recibido un apoyo explícito y claro del departamento:

P3: Pero sí, el departamento apoya todo (...) Yo con el departamento no tuve, en ese aspecto problemas. El resto del departamento está muy de acuerdo con estas prácticas."

Pero la percepción de la falta de apoyo vuelve a emerger cuando el análisis se sitúa a nivel de centro. Falta de apoyo explícito que, de nuevo, se manifiesta en forma de aislamiento y se expresa con cierto tono de resignación:

P4."Intentar aplicar innovación desde un punto de vista aislado como manchas de aceite que contaminan una organización tan compleja (...) una división tan importante como la nuestra, es que es una utopía, . un intento es un intento pero que la dificultad se produce cuando estás absolutamente aislado."

Ciertamente, la percepción de estos docentes no describe una realidad nueva. Si hay algún rasgo típico de la docencia en la universidad, es el del individualismo y aislamiento de los docentes. El trabajo docente es una actividad secularmente individual y de puertas adentro como pocas otras ocupaciones. Los docentes pueden encontrar en el aislamiento ciertas ventajas como la seguridad psicológica que puede representar saberse dueño absoluto y exclusivo de una situación y no estar sometido al escrutinio directo ni inmediato de ninguna otra persona. Sin embargo, sa falta de diálogo y comunicación entre docentes supone un obstáculo para la mejora del diseño curricular, de la enseñanza y del fomento del aprendizaje del alumnado.

El aislamiento e individualismo no parecen ser las únicas formas que adoptan las relaciones que mantienen entre sí el profesorado:

P4. "El departamento está muy organizado por cátedras. Es decir, cátedras que no se dan razones la una a la otra, sino que toman sus propias decisiones."

Esta cita permite ilustrar otra de las formas que adopta la cultura universitaria: la balcanización. Esta forma de interacción se produce cuando en los departamentos aparecen grupos separados de docentes con intereses y concepciones disciplinares y docentes diferenciadas y que, en ocasiones, rivalizan y compiten por posiciones de privilegio y preferencia.

Las diversas formas que, como hemos visto, adopta la cultura profesional dominante en la universidad, no sólo pueden constituir rémoras puntuales a la innovación, sino que constituyen el principal freno endógeno para la adopción de una nueva cultura institucional que tome la colaboración como su eje estructurante. En efecto, la fuerza del ambiente, las rutinas diarias, la inercia de los comportamientos, la presión de las expectativas sociales y las relaciones de poder-dominación entre las diversas jerarquías docentes hacen que las expectativas y funciones de los docentes noveles se acomoden sin debate previo ni deliberación reflexiva a los ritmos y rutinas habituales de las prácticas tradicionales docentes en la universidad:

P8. "Es un dilema que tengo yo: qué hago que memoricen o que no memoricen, que se anden con apuntes o sin apuntes (...) pensar en mi departamento sin apuntes es imposible. (...) Es que no hay nadie que lo haga, yo soy el último mono del departamento, (...) si digo: valoremos la posibilidad, pondrían el grito en el cielo." 


\section{e) Las defensas del alumnado ante la innovación docente}

En el seno de esta cultura individualista, jerárquica y transmisiva, el papel que interioriza el alumno y las expectativas que tiene acerca de la actuación docente y de la finalidad de la docencia universitaria inhiben o dificultan las propuestas de cambio sugeridas por el profesor novel:

P7. "Yo veo también un problema que tiene que ver a como están acostumbrados los alumnos a trabajar. Tú llegas y les dices que les has cambiado el sistema de estudio y de preparar la asignatura...ellos están acostumbrados a tener unos buenos apuntes, y saben que con aquellos van a ir al examen y van a aprobar, etc.(...) Vas a ir con innovación y tienes una baja respuesta de seguimiento, y desincentiva un poco lo que es esta voluntad dinámica de llevar las cosas a cabo."

El profesorado novel intenta llevar a la práctica una docencia que fomente el aprendizaje significativo y las habilidades de pensamiento, pero esas pretensiones no son compartidas por el alumnado ya que la cultura institucional dominante no las exige ni fomenta:

P7.: "En este caso estamos en un modelo de universidad totalmente escolar. Esencialmente en su chip interno tienen que ver la carrera como una sucesiva progresión de superación de exámenes".

\section{CONCLUSIONES}

Los cambios introducidos en la práctica docente por el profesorado novel tras su participación en el postgrado, ofrecen evidencias del impacto del curso y de la transferibilidad de los contenidos impartidos a la realidad de los participantes. Parece que el docente novel, tras participar en el curso, haya adoptado una actitud más crítica y reflexiva ante su propia práctica y ante la tradición docente dominante en su facultad. Por lo tanto, el curso ha ofrecido al docente novel argumentos suficientes para teorizar sobre su propia práctica, para comprenderla con mayor profundidad y conseguir una clarificación conceptual de la docencia universitaria.

Conseguir una comprensión más elaborada de la docencia universitaria, ha sido el origen de los procesos de cambio que el docente ha llevado a cabo en el aula. Al hablar de cambios en la conceptualización de la docencia, nos referimos a cambios en la concepción papel del profesor, finalidad de la enseñanza, rol del alumno, comprensión de los procesos de enseñanza-aprendizaje y relación profesoralumno. Es importante señalar la relación entre estos elementos y cómo los cambios en uno de ellos influyen en todos los demás. Según los comentarios del profesorado novel, parece ser que el curso de postgrado les ha ayudado a conseguir una comprensión más globalizada de la enseñanza universitaria. El docente novel, tras participar en el postgrado, percibe el aprendizaje œmo un proceso individual que se produce en interacción con el medio. Según el profesorado novel, la relación entre la enseñanza y el aprendizaje no es lineal o causal, es decir el aprendizaje no depende directamente de la enseñanza recibida por el profesor, sino que el responsable último del proceso de aprendizaje es el alumnado. No obstante, el docente novel entiende que aquello que un individuo puede llegar a aprender depende del tipo y grado de ayuda que recibe. Por ello, perciben al docente universitario como mediador del aprendizaje del alumno más que como un transmisor de contenidos. Entienden que la enseñanza debe pasar de la explicación y la reproducción de conocimientos a la investigación y la construcción de aprendizajes significativos. Por ello su función en el aula no se reduce ya a la transmisión de contenidos académicos, sino que intentan generar y crear contextos ricos en oportunidades de aprendizaje. 
Para los profesores noveles, el contexto institucional y social en el que desempeñan su labor docente ha constituido, en ocasiones, un freno al cambio y a las innovaciones que estaban tratando de desarrollar. Algunos de estos obstáculos, dada su naturaleza, van más allá de la capacidad y/o posibilidad del profesor para superarlos. Son factores externos al docente novel, aspectos propios del contexto institucional y que, además, influyen en su motivación e interés por la innovación y la calidad de su labor docente. De la opinión de los docentes noveles se deduce que aquellas instituciones universitarias cuyos profesores están comprometidos con el cambio educativo y la mejora docente cuentan, entre otras cosas, con recursos adecuados y suficientes, apoyo y reconocimiento institucional a la innovación y una cultura académica basada en la colaboración.

La existencia de un déficit de recursos materiales disponibles es, según el profesorado novel, un factor inhibitorio para el cambio y la mejora en la docencia universitaria. El elevado número de alumnos por asignatura es otra de las dificultades que, según el docente novel, dificulta el desarrollo de innovaciones en el aula. Esta masificación comporta, por otra parte, una elevada dedicación. Ese aumento de tiempo de dedicación que implica realizar una innovación educativa ha sido también señalado por el docente novel como un factor que dificulta la introducción de cambios.

Uno de los aspectos más comentados por el docente novel es la inseguridad que perciben al iniciar su práctica docente. Ya se ha señalado que esta inseguridad es consecuencia de la falta de experiencia y conocimiento pedagógico del profesor novel. Este rasgo, fácilmente identificable en aquellos que se inician en la docencia universitaria, es un indicio para proponer y consolidar prácticas formativas encaminadas a reforzar la actuación inicial del docente. Los programas de inducción a la docencia junto con el apoyo al docente son una buena solución para reducir la inseguridad y la falta de confianza que el docente novel experimenta.

Los docentes noveles se encuentran en facultades con una cultura académica individualista y poco dada al trabajo cooperativo entre el profesorado. Los participantes en el postgrado manifiestan haberse sentido solos en los procesos de innovación desarrollados en su práctica docente, y comentan que no han percibido ningún tipo de reconocimiento por parte del departamento al que pertenecen.

En síntesis, la elevada variabiliad de las dificultades que encuentran los profesores noveles no se debe tanto a sus características personales y su perfil profesional como al contexto académico, institucional y cultural en el que se sitúa la innovación.

Las dificultades para el desarrollo de innovaciones didácticas no surgen solamente del contexto institucional en el que el docente realiza su labor. En ocasiones, el alumnado frena las iniciativas de cambio propuestas por el docente novel. Esto se debe al rol que el alumnado ha interiorizado como consecuencia de pertenecer a un contexto institucional con una cultura fundamentalmente individualista, jerárquica y transmisiva. Las expectativas que el alumnado ha ido construyendo acerca de su papel como estudiante dificultan la realización de actividades que requiera su participación activa y en parte autónoma en el proceso de aprendizaje. Los alumnos muestran, en ocasiones, una cierta predisposición hacia el aprendizaje memorístico y academicista. Por ello, reclaman al docente universitario una enseñanza tradicional que no vaya más allá de la reproducción de contenidos teóricos. Nótese, sin embargo, que el alumnado ha valorado, de manera positiva, aquellas innovaciones introducidas por el docente novel en el aula. Se deduce por lo tanto, que el rechazo del alumno hacia el cambio es inicial, y producto de su 
pertenencia a una institución con una cultura docente de carácter tradicional. Tras participar en una actividad innovadora, los alumnos valoran, en gran medida, otras formas de aprendizaje, a la vez que redefinen el rol del profesor y la finalidad de la docencia universitaria.

\section{REFERENCIAS BIBLIOGRÁFICAS}

ÁlvarEZ, V., GarCíA, E. Y GIL, J. La calidad de la enseñanza universitaria desde la persepectiva de los profesores mejor valorados por los alumnos. Revista de Educación (319), (1999), 273-290.

Angulo RAsco, F. De la investigación sobre la enseñanza al conocimiento docente. In A. PÉrez GómeZ, A. BARQUIN, J Y ANGULO RASco, F.(EDS.), Desarrollo profesional del docente. Política, investigación y práctica. (pp. 261-319). Madrid: AKAL Textos, 1999.

ELLIOTT, J. Reconstructing Teacher Education. Teacher Development. Londres: Falmer Press, 1993.

ESCUDERO MuÑOZ, J. M. La innovación educativa en tiempos turbulentos. Cuadernos de Pedagogía(240), (1995). 18-21.

Feiman Nesmer, S. Teacher Preparation: Structural and Conceptual Alternatives. En R. Houston (Ed.), Handbook of research of teacher education (pp. 212-233). Nueva York: MacMillan, 1990.

FULLAN, M. The new meaning of educational change. Chicago: Teacher College Press, 1991.

GEERTZ, C. La interpretación de las culturas. Barcelona: Gedisa, 1987. Primera edición publicada en 1973.

GLASER, B., Y StRAuSS, A. The discovery of Grouded Theory. Chicago: Aldine, 1967.

KILPATRICK. D.L. Evaluación de acciones formativas. EPISE: Barcelona. 1999.

Medina MoyA, J. L. La Pedagogía del Cuidado: saberes y prácticas en la formación universitaria en Enfermería. Barcelona: Laertes, 1999.

PÉReZ GómeZ, A. El Pensamiento Práctico del Profesor: Implicaciones en la Formación del Profesorado. In A. Villa (Ed.), Perspectivas y problemas de la función docente II (pp. 128-148). Madrid: Narcea, 1988. 


\title{
Contactar
}

Revista lberoamericana de Educación

\author{
Principal OEI
}

